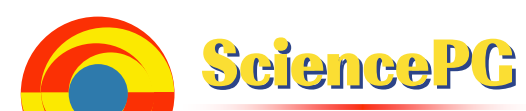

\section{Hybrid method for ciphering colored image}

\author{
Salman Abd Kadum, Ali Abdul Azeez Mohammad Baker \\ Computer Systems Techniques, Al Furat Al Wast University, Al Najaf Technical Institute, Al Najaf, Iraq.
}

\section{Email address:}

salman_abd_2002@yahoo.com (S. A. Kadum), alia.qazzaz@uokufa.edu.iq (A. A. A. M. Baker)

\section{To cite this article:}

Salman Abd Kadum, Ali Abdul Azeez Mohammad Baker. Hybrid method for Ciphering Colored Image. Automation, Control and Intelligent Systems. Special Issue: Artificial Nano Sensory System. Vol. 3, No. 2-1, 2015, pp. 22-34. doi: 10.11648/j.acis.s.2015030201.15

\begin{abstract}
Security has more and more importance in current era especially in the organizations where information is more critical and more effective, so there are many methods that will be used in keeping information secure and safety like steganography, cryptography, barcode, password, and biometrics. different techniques will be used to protect image data from unauthorized access, In this paper a hybrid method will be proposed for ciphering secure image by translating information from original $\mathrm{RGB}$ image to $\mathrm{YC}_{\mathrm{b}} \mathrm{C}_{\mathrm{r}}$ format then using public key algorithm after normalized pixels values to insure that will be within public and prevent keys region after that the Modified Arnold Transform, Generalized Fibonacci Transform, and Fibonacci-Lucas Transform will be applied on each ciphered band from $\mathrm{YC}_{\mathrm{b}} \mathrm{C}_{\mathrm{r}}$ image respectively.
\end{abstract}

Keywords: Cryptography, Ciphering, Deciphering, Color image, Public Key, Private Key, Arnold Transform, Fibonacci Transform, Fibonacci-Lucas Transform

\section{Introduction}

Cryptography is the science of hiding information, or is a fundamental tool for the protection of sensitive information. Encryption is a way of talking to someone while other people are listening, but such that the other people cannot understand what you are saying. It can also be used to protect data in storage. The goal of cryptography is to make data unreadable by unwanted persons. According to the key that will be used in ciphering algorithm, Cryptography algorithms are divided into two types, symmetric key and public-key (asymmetric) algorithms. Symmetric algorithms are used the same key in both ciphering and deciphering algorithms. While Public-key encryption algorithms used a pair of keys, the first key is used in encryption information that will be sent to a receiver which is named (public) or the second key who owns the corresponding private key which is used in decryption the information. Image encryption techniques are used to overcome the problem of secure transmission for both images and text over the electronic media by using the suitable cryptographic algorithms.

\subsection{Public Key Cryptography}

Public key algorithm is a powerful method for ciphering information, and the strength of this algorithm depends on the strength of the public and private keys. As we know that every person who wants to send secret information by using unsecured channel must used a suitable way to protect this information, one of the most important protection method which is public key algorithm.

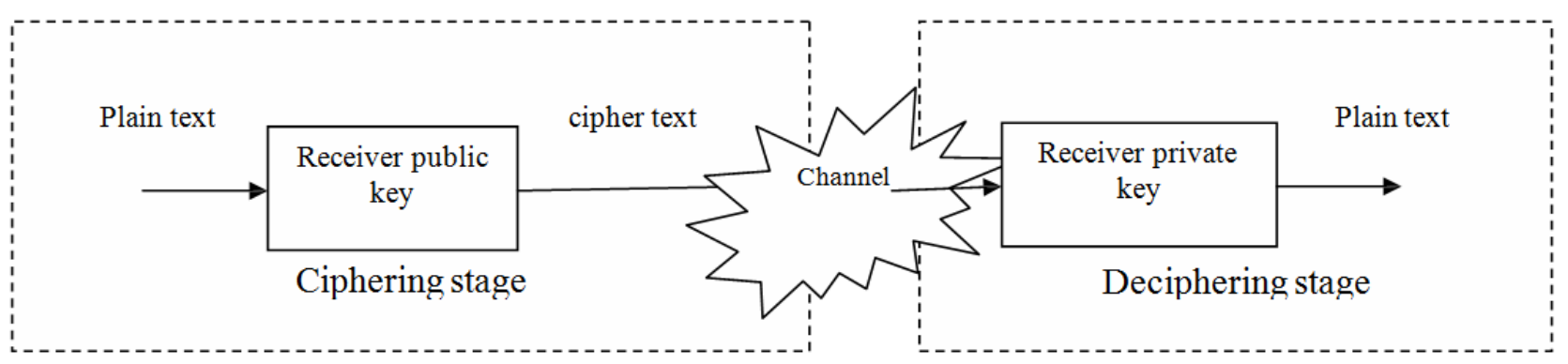

Figure (1). Public key encryption method. 


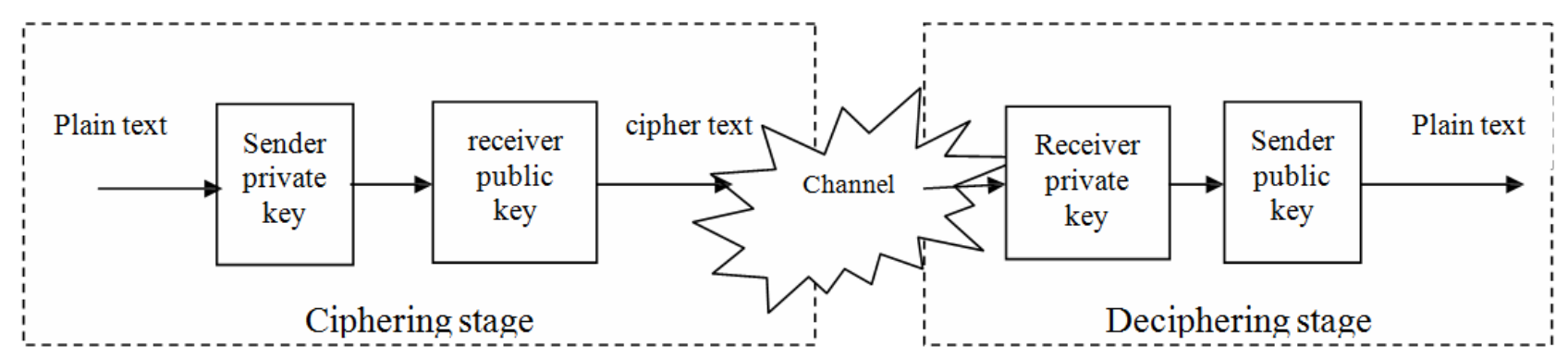

Figure (2). Signature encryption method.

Every person has two related keys (public and private), and if any person want to send secrete message to another person, he must used the public key of the received person to cipher the message and then send it by using any transform media, after that the received person must be deciphering the message to extract the information, and this operation done by using receiver private key as illustrated in figure (1), in some cases sender ciphering the message by using sender private key firstly then receiver public key and this state called the signature, so the receiver deciphering the message by using receiver private key firstly, then sender public key to extract information as illustrated in figure(2).

The two keys of any person are related and they created from primary numbers by using special method as follows:

- Selecting two big prime numbers (p, q), and big number (e) randomly.

- Calculating $\theta(n)=(p-1) \times(q-1), n=p \times q$.

- Calculating $d=\frac{G C D((p-1),(q-1)) \times \theta(n)+1}{e}$.

- Constructing public key (e, n), and private key (d, n).

For example if person1 wants to construct his keys, firstly he chooses $p=23, q=17, e=15$.

$$
\begin{gathered}
\theta(n)=(23-1) \times(17-1)=352, n=23 \times 17=391 \\
d=\frac{G C D(22,16) \times 352+1}{15}=\frac{705}{15}=47
\end{gathered}
$$

Then the public key of person1 is $(15,391)$, and the private key of person1 is $(47,391)$.

For ciphering any plain text by using public key algorithm the equation (1) will be used as follows:

$$
C_{(\text {number })}=(\text { number })^{e r} \bmod n_{r}
$$

Where $\left(e r, n_{r}\right)$ is the receiver public key.

For example if any person wants to send message (HI) to person1 with above keys then,

$$
\begin{gathered}
\operatorname{ASCII}(H)=72, \operatorname{ASCII}(I)=73 \\
C(H)=(72)^{15} \bmod 391=81 \\
C(H)=(73)^{15} \bmod 391=279
\end{gathered}
$$

Therefore the person sends the message 81,279 to person 1 .
For deciphering any cipher text by using public key algorithm the equation (2) will be used as follows:

$$
P_{(\text {number })}=(\text { number })^{d r} \bmod n_{r},
$$

Where $\left(\mathrm{dr}, \mathrm{n}_{\mathrm{r}}\right)$ is the receiver private key.

For the above cipher text that received by person1, he will extract plain text by applying equation (2) as follows:

$$
\begin{aligned}
& P_{(81)}=(81)^{47} \bmod 391=72=H \\
& P_{(81)}=(279)^{47} \bmod 391=73=I
\end{aligned}
$$

Plain text is $(H I)$

\subsection{Arnold Transform}

It is a transform $\Gamma: T^{2} \rightarrow T^{2}$

$$
\left[\begin{array}{l}
x^{\prime} \\
y^{\prime}
\end{array}\right]=\left[\begin{array}{ll}
2 & 1 \\
1 & 1
\end{array}\right]\left[\begin{array}{l}
x \\
y
\end{array}\right][\bmod N]
$$

Where $x, y, x^{\prime}, y^{\prime} \in\{0,1,2, \ldots N-1\}$, and $\mathrm{N}$ is the size of an original squared image.

A new image will be produced when all points in the original image are ciphered by equation (1). The Arnold transform change the position of each pixel in original image but the value of this pixel stay with same original value, this transform is simple but powerful which is very much easy in spatial domain.

The security level of the encrypted image becomes danger when it is encrypted by using the basic Arnold transform as it depend on a single $2 \times 2$ array so, to enhance the security level of the encrypted image the basic Arnold transform is modified as follows:

$$
\left[\begin{array}{l}
x^{\prime} \\
y^{\prime}
\end{array}\right]=\left[\begin{array}{cc}
k+1 & k \\
1 & 1
\end{array}\right]\left[\begin{array}{l}
x \\
y
\end{array}\right][\bmod N]
$$

Where $x, y, x^{\prime}, y^{\prime} \in\{0,1,2, \ldots N-1\}, \mathrm{N}$ is the size of a digital squared image, and $k=\{0,1,2,3, \ldots\}$

Unlike equation (1) where there is a single map, equation (4) provides a new map for each value of $\mathrm{k} \mathrm{so}$; the security level of the scrambled image against attack (trial decryption 
by an unauthorized user) will be increased.

For $k=1$, equation (4) is same as the original Arnold transform of equation (1).

\subsection{Fibonacci Transform}

This is a special case of the basic Arnold transform, the equation of the transform is:

$$
\left[\begin{array}{l}
x^{\prime} \\
y^{\prime}
\end{array}\right]=\left[\begin{array}{ll}
1 & 1 \\
1 & 0
\end{array}\right]\left[\begin{array}{l}
x \\
y
\end{array}\right][\bmod N]
$$

Generally, the Fibonacci sequence $F_{n}$, is a sequence of integer numbers that given by equation (6):

$$
F_{n}=\left\{\begin{array}{lr}
0 & n=0 \\
1 & n=1 \\
F_{n-1}+F_{n-2} \text { otherwise }
\end{array}\right.
$$

By applying equation (6) the Fibonacci series constitutes the numbers:

$$
0,1,1,2,3,5,8,13,21,34,55,89,144,233 \ldots
$$

It can be easily seen that a $2 \times 2$ matrix formed by any four consecutive terms of the Fibonacci Numbers is a matrix and can be considered as an image scrambler. A generalized Fibonacci Transform can be defined as a mapping $\Gamma: T^{2} \rightarrow T$ 2 such that:

$$
\left[\begin{array}{l}
x^{\prime} \\
y^{\prime}
\end{array}\right]=\left[\begin{array}{cc}
F_{i} & F_{i+1} \\
F_{i+2} & F_{i+2}
\end{array}\right]\left[\begin{array}{l}
x \\
y
\end{array}\right][\bmod N]
$$

Where, $x, y, x^{\prime}, y^{\prime} \in\{0,1,2 \ldots N-1\}, F^{i}$ is the ith term of the Fibonacci Series. And $\mathrm{N}$ is the zero size of a digital square image.

\subsection{Lucas Series}

Lucas series is a special case of Fibonacci series can be defined as follows:

$$
F_{n}=\left\{\begin{array}{lr}
2 & n=0 \\
1 & n=1 \\
L_{n-1}+L_{n-2} \text { otherwise }
\end{array}\right.
$$

By applying equation (8) the Lucas series constitutes the numbers:

$$
2,1,3,4,7,11,18,29,47,76,123,199,322,521 \ldots
$$

Unlike the Fibonacci series, the terms of the Lucas series does not form a periodic map and cannot be used for image encryption but by combining the terms of the $N$ Fibonacci and Lucas series, a series of new periodic maps will be formed which can be used for image scrambling.

\subsection{Fibonacci-Lucas Transform}

The equation of this transform is:

$$
\left[\begin{array}{l}
x^{\prime} \\
y^{\prime}
\end{array}\right]=\left[\begin{array}{ll}
F_{1} & F_{i+1} \\
L_{i} & L_{i+1}
\end{array}\right]\left[\begin{array}{l}
x \\
y
\end{array}\right][\bmod N]
$$

Where, $x, y, x^{\prime}, y^{\prime} \in\{0,1,2 \ldots N-1\}, F^{i}$ is the ith term of the $F 12$ series, $L^{i}$ is the ith term of the Lucas series, and $N$ is the size of a digital squared image.

Continuing in this way we can form an infinitely many transforms and just like the Modified Arnold and Fibonacci Transforms, all of these matrices will be periodic in nature with a maximum possible periodicity of $N^{2}-1$.

\section{The Proposed System}

The proposed system consist of two stages, the first stage for encryption information and the second one for decryption, each one of these stages consist of many steps. The encryption steps will be illustrated in figures (3), which are:

- Translate image into $Y C_{b} C_{r}$ format and separating colored image into three grayscale image, each one of them represent one band like y, $C_{b}, C_{r}$.

- Applying normalization process for all pixels in each band according to the key value.

- Ciphering each pixel in first grayscale image ( $y$ band) by using receiver public key.

- Ciphering each pixel in second grayscale image $\left(C_{b}\right.$ band) by using receiver public key and sender private key.

- Ciphering each pixel in third grayscale image $\left(C_{r}\right.$ band) by using received public key.

- Scrambling all pixels in first band by using Arnold transform

- Scrambling all pixels in second band by using Fibonacci transform.

- Scrambling all pixels in third band by using FibonacciLucas transform

- Reconstructing colored image.

- Scrambling all pixels in colored by using new Arnold transform.

While the decryption steps illustrated in figure (4), which are:

- Applying inverse Arnold transform on colored image.

- Separating ciphered image into three grayscale image (bands).

- Applying inverse Arnold transform on first band.

- Applying inverse Fibonacci transform on second band.

- Applying inverse Fibonacci-Lucas transform on third band.

- Deciphering each pixel in first grayscale image by using receiver private key.

- Deciphering each pixel in second grayscale image by using receiver private key and sender public key.

- Deciphering each pixel in third grayscale image by using received private key.

- Return original values before normalization.

- Reconstructing $Y C_{b} C_{r}$ image.

- Translate to original .BMP image. 


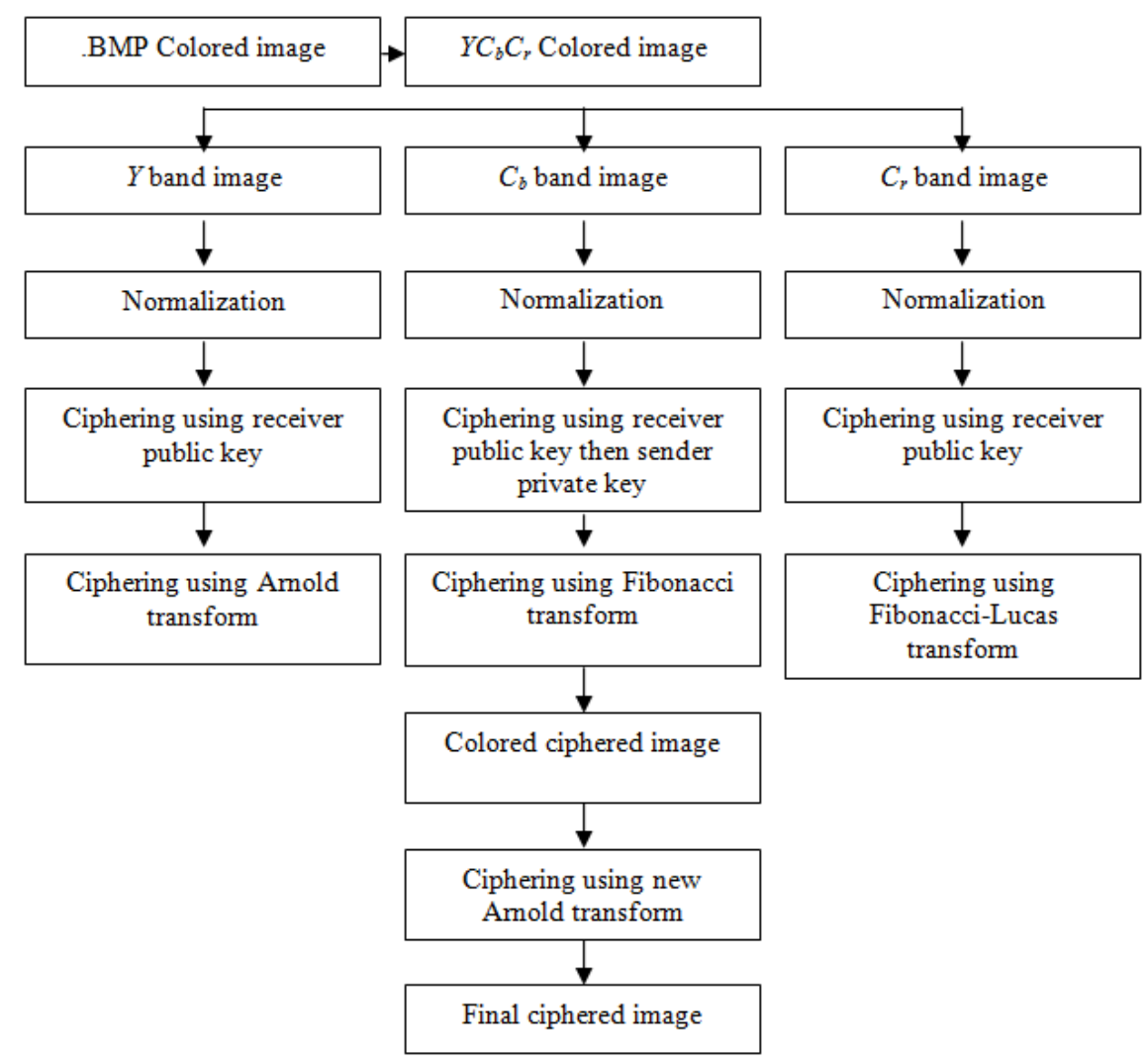

Figure (3). Encryption stage of the proposed system.

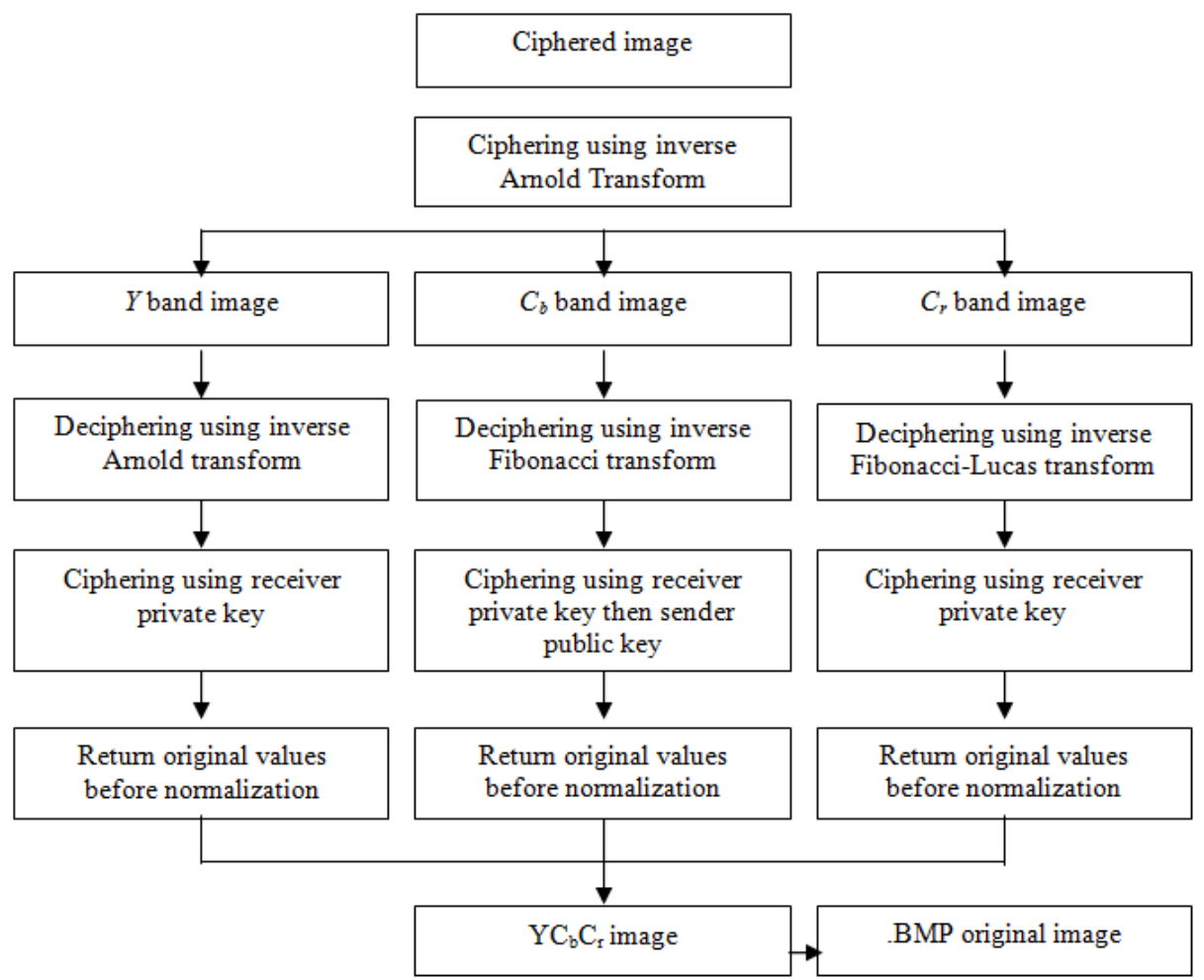

Figure (4). Decryption steps of the proposed system.

Each step in the proposed system will be illustrated with an example, by using the following keys:

- receiver public key $(3,187)$,
- receiver private key $(107,187)$,

- sender public key $(11,133)$, and

- sender private key $(59,133)$. 
With the following bands for part of colored image as illustrated in figure (5).

\begin{tabular}{|c|c|c|}
\hline 3 & 200 & 210 \\
\hline 30 & 100 & 250 \\
\hline 40 & 150 & 255 \\
\hline
\end{tabular}

a. red band

\begin{tabular}{|c|c|c|}
\hline 150 & 80 & 100 \\
\hline 22 & 66 & 120 \\
\hline 8 & 78 & 250 \\
\hline
\end{tabular}

b. green band

\begin{tabular}{|c|c|c|}
\hline 250 & 150 & 30 \\
\hline 100 & 40 & 20 \\
\hline 90 & 20 & 10 \\
\hline
\end{tabular}

c. blue band

Figure (5). Bands for part of colored image.

\section{First Stage}

This stage for encryption information contains many steps as follow:

\subsection{Translating to $\mathrm{YC}_{b} C_{r}$ Image}

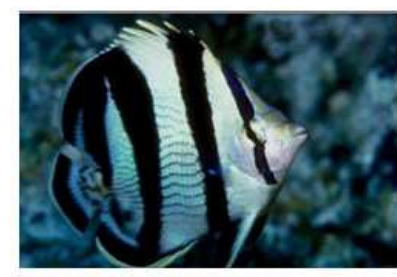

a-BMP image

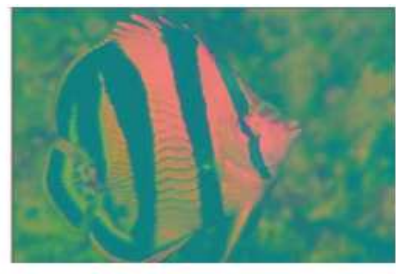

b- $Y C_{b} C_{r}$ image
Figure (6). Translating to $Y C_{b} C_{r}$ image.
$\left(Y C_{b} C_{r}\right)$ image will be created from BMP image by using equation (10) as illustrated in figure (6) and (7):

$$
\left[\begin{array}{c}
Y \\
C_{b} \\
C_{r}
\end{array}\right]=\left[\begin{array}{c}
16 \\
128 \\
128
\end{array}\right]+\left[\begin{array}{ccc}
0.299 & 0.587 & 0.114 \\
-0.168 & -0.331 & 0.5 \\
0.5 & -0.418 & -0.081
\end{array}\right] \times\left[\begin{array}{c}
\text { red } \\
\text { green } \\
\text { blue }
\end{array}\right]
$$

To calculate values of all pixels in each band from red, green, and blue bands, Equation (10) will be applied as follows:

For y band: $16+(0.299 \times$ red $+0.587 \times$ green $+0.114 \times$ blue $)$. For $\mathrm{Cb}$ band: $128+(-0.168 \times$ red- $0.331 \times$ green $+0.5 \times$ blue $)$. For Cr band: $128+(0.5 \times$ red- $0.418 \times$ green- $0.081 \times$ blue $)$.

The results of applying these states will be applied in figure (7).

\begin{tabular}{|l|l|l|}
\hline 133 & 140 & 141 \\
\hline 49 & 89 & 163 \\
\hline 43 & 109 & 240 \\
\hline
\end{tabular}

\begin{tabular}{|l|l|l|}
\hline 203 & 143 & 75 \\
\hline 173 & 109 & 56 \\
\hline 164 & 87 & 7 \\
\hline
\end{tabular}

\begin{tabular}{|l|l|l|}
\hline 47 & 182 & 189 \\
\hline 126 & 147 & 201 \\
\hline 137 & 169 & 150 \\
\hline
\end{tabular}

Figure (7). $Y C_{b} C_{r}$ image values.

\subsection{Normalization}

Normalization process is an important step to prepare image pixels for ciphering because we cannot apply modulo of (133) on 256 values without loose information, so all values must be less than (133) to insure information retrieve, these process applied on all bands according to value of (n) in the key. The y band will be encrypted by key $(3,187)$ so all pixels in y band must have values between 0 and 186, in the same way blue band will be encrypted with key $(3,187)$ so all pixels in $\mathrm{Cr}$ band must have values between 0 and 186, while the $\mathrm{Cb}$ band will be encrypted firstly by key $(3,187)$ so all pixels in y band must have values between 0 and 186, after that the ciphered pixels in $\mathrm{Cb}$ band will be ciphered by key $(59,133)$ so all pixels in ciphered $\mathrm{Cr}$ band must have values between 0 and 132 .

For y band: $133 \times 186 / 255=97,140 \times 186 / 255=102$, and so on for other values. For $\mathrm{Cr}$ band: $47 \times 132 / 255=24$, $182 \times 132 / 255=94$, and so on for other values.

And for $\mathrm{Cb}$ band: the first normalized process is $203 \times 186 / 255=148,143 \times 186 / 255=104$.

The second normalized process will be done after ciphering with key $(3,187)$. The pixels value of colored image in figure (7) will be normalized as illustrated in figure (8).

The results of applying this step will be illustrated in figure (9).

\begin{tabular}{|l|l|l|}
\hline 97 & 102 & 103 \\
\hline 36 & 65 & 110 \\
\hline 31 & 80 & 175 \\
\hline
\end{tabular}

a. $Y$ band

\begin{tabular}{|l|l|l|}
\hline 148 & 104 & 55 \\
\hline 126 & 80 & 41 \\
\hline 120 & 63 & 5 \\
\hline
\end{tabular}

b. $C_{b}$ band

\begin{tabular}{|l|l|l|}
\hline 24 & 94 & 98 \\
\hline 65 & 76 & 104 \\
\hline 71 & 87 & 78 \\
\hline
\end{tabular}

c. $C_{r}$ band

Figure (8). Bands of normalization Image. 


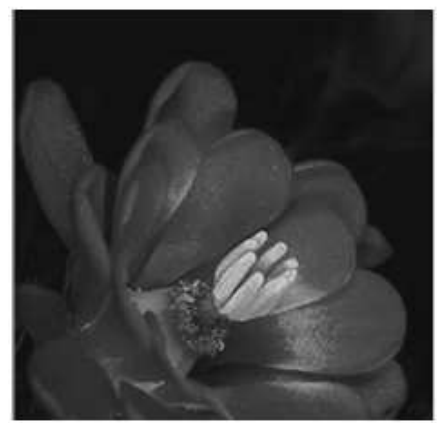

a- original $Y$ band

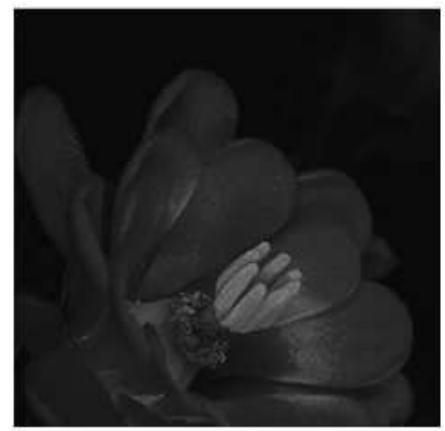

d- $Y$ band after normalization

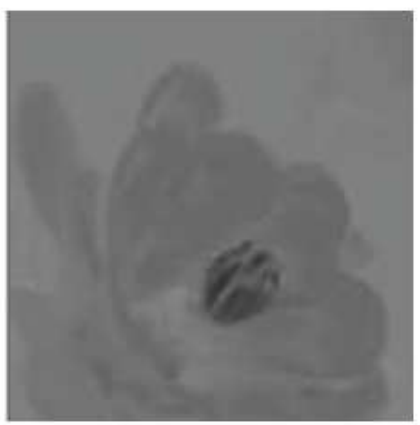

b- original $C_{b}$ band

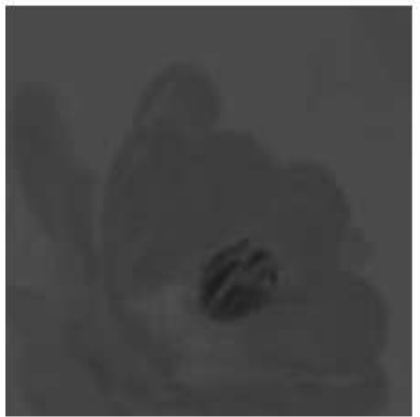

e- $C_{b}$ band after normalization

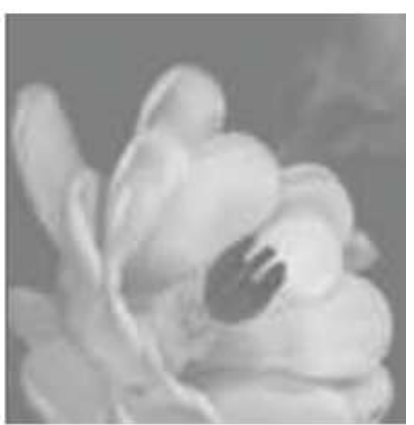

c- original $C_{r}$ band

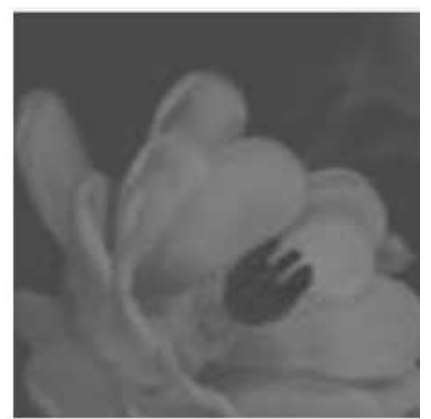

e- $C_{r}$ band after normalization

Figure (9). Results of normalization step.

\subsection{Public Key Encryption}

As illustrated in the proposed system block diagram, each band will be ciphered with different key or keys, so this step will be separated into three states.

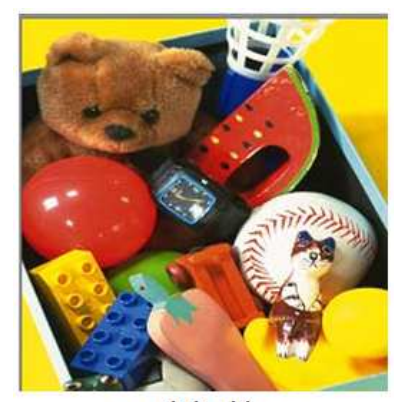

a- original image

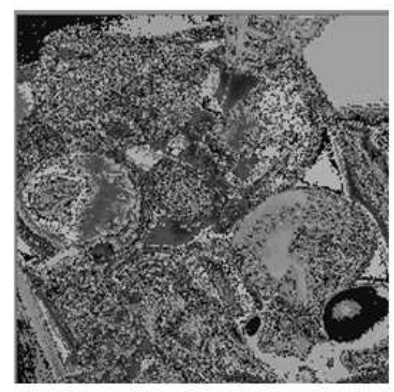

b- ciphered $Y$ image
Figure (10). Encryption Y image.

$C(97)=(97)^{3} \bmod 187=113$

\begin{tabular}{|l|l|l|}
\hline 97 & 102 & 103 \\
\hline 36 & 65 & 119 \\
\hline 31 & 80 & 175 \\
\hline
\end{tabular}

a. original image

\begin{tabular}{|l|l|l|}
\hline 113 & 170 & 86 \\
\hline 93 & 109 & 102 \\
\hline 58 & 181 & 142 \\
\hline
\end{tabular}

Figure (11). Results after applying key $(3,187)$ on Y image.

\subsection{1. $Y$ Band}

In this band, the cipher used received public key for encryption all pixels in this band by applying equation (1) as illustrated in figure (10) as well as in calculating the new ciphered values for supposed example with key $(3,187)$ as shown in figure (11).

\subsection{2. $C_{b}$ Band}

In this band, the cipher used received public key and sender private key for encryption all pixels in this band by applying equation (1) with additional normalization process as illustrated in figure (12) as well as in calculating the new ciphered values for supposed example with keys $(3,187)$ and $(59,133)$ as shown in figure $(13)$.

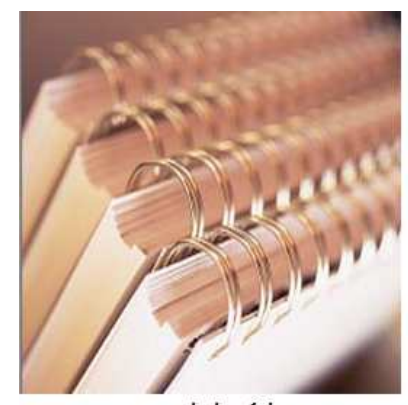

a- original image

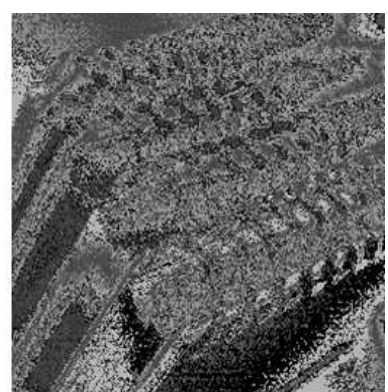

b- ciphered $C_{b}$ image
Figure (12). Encryption $C_{b}$ image.

$C(97)=(148)^{3} \bmod 187=147$ then normalized all values to cipher with key $(59,133)$

$$
147 \times(132 / 255)=76
$$

$$
C(76)=(76)^{59} \bmod 133=76
$$




\begin{tabular}{|l|l|l|}
\hline 148 & 104 & 55 \\
\hline 126 & 80 & 41 \\
\hline 120 & 63 & 5 \\
\hline
\end{tabular}

a. original image

\begin{tabular}{|l|l|l|}
\hline 147 & 59 & 132 \\
\hline 37 & 181 & 105 \\
\hline 120 & 28 & 125 \\
\hline
\end{tabular}

b. first ciphered image

\begin{tabular}{|l|l|l|}
\hline 76 & 31 & 98 \\
\hline 19 & 94 & 54 \\
\hline 62 & 14 & 65 \\
\hline
\end{tabular}

c. normalized image

\begin{tabular}{|l|l|l|}
\hline 76 & 103 & 45 \\
\hline 38 & 75 & 80 \\
\hline 104 & 105 & 88 \\
\hline
\end{tabular}

c. final ciphered image

Figure (13). Results ciphering $C_{b}$ image.

\subsection{3. $C_{r}$ Band}

In this band, the cipher used sender private key for encryption all pixels in this band by applying equation (1) as illustrated in figure (14) as well as in calculating the new ciphered values for supposed example with key $(59,133)$ as shown in figure (15).

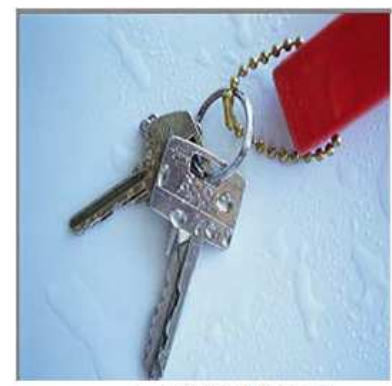

a- original image

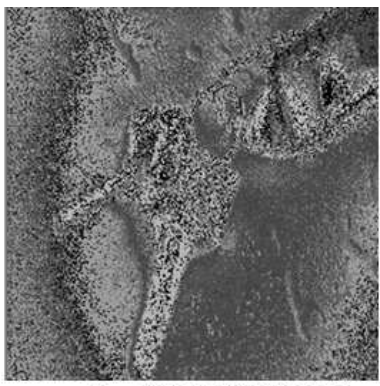

b- ciphered $\mathrm{Cr}$ image
Figure (14). Encryption $C_{r}$ image.

\begin{tabular}{|l|l|l|}
\hline 97 & 102 & 103 \\
\hline 36 & 65 & 119 \\
\hline 31 & 80 & 175 \\
\hline
\end{tabular}

a. original image

\begin{tabular}{|l|l|l|}
\hline 113 & 170 & 86 \\
\hline 93 & 109 & 102 \\
\hline 58 & 181 & 142 \\
\hline
\end{tabular}

b. ciphered $C_{r}$ image
Figure (15). Results after applying key $(3,187)$ on $C_{r}$ image.

\subsubsection{Scrambling Pixels in Each Band}

As illustrated in proposed system block diagram, each band will be scrambled with specific transform, so this step will be separated into three states.

\subsubsection{Y Band}

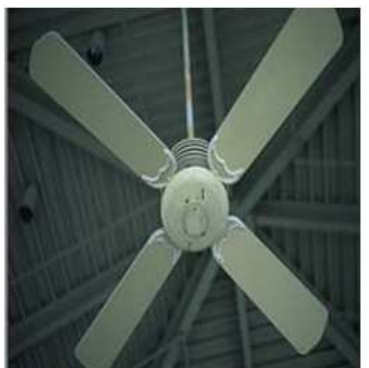

a- original image

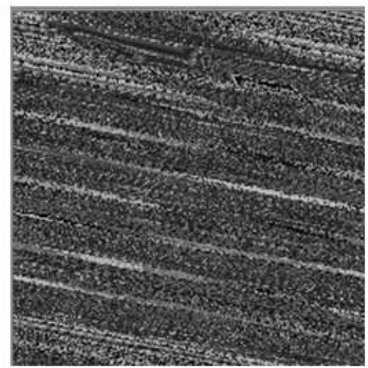

b- ciphered $Y$ image
In this band, the cipher used Arnold transform for scrambling all pixels by applying equation (4) as illustrated in figure (16) as well as in calculating the new ciphered values for supposed example as shown in figure (17).

$$
\begin{aligned}
& {\left[\begin{array}{l}
x^{\prime} \\
y^{\prime}
\end{array}\right]=\left[\begin{array}{ll}
6 & 5 \\
1 & 1
\end{array}\right]\left[\begin{array}{l}
0 \\
0
\end{array}\right][\bmod 3]=(0,0),} \\
& {\left[\begin{array}{l}
x^{\prime} \\
y^{\prime}
\end{array}\right]=\left[\begin{array}{ll}
6 & 5 \\
1 & 1
\end{array}\right]\left[\begin{array}{l}
0 \\
1
\end{array}\right][\bmod 3]=(2,1)}
\end{aligned}
$$

\begin{tabular}{|l|l|l|}
\hline 97 & 102 & 103 \\
\hline 36 & 65 & 119 \\
\hline 31 & 80 & 175 \\
\hline
\end{tabular}

a. original image

\begin{tabular}{|l|l|l|}
\hline 97 & 36 & 31 \\
\hline 119 & 175 & 103 \\
\hline 80 & 170 & 65 \\
\hline
\end{tabular}

b. ciphered $Y$ image
Figure (17). Results after scrambling Y image.

\subsubsection{2. $C_{b}$ Band}

In this band, the cipher used Fibonacci transform for scrambling all pixels in this band by applying equation (7) as illustrated in figure (18) as well as in calculating the new ciphered values for supposed example as shown in figure (19).

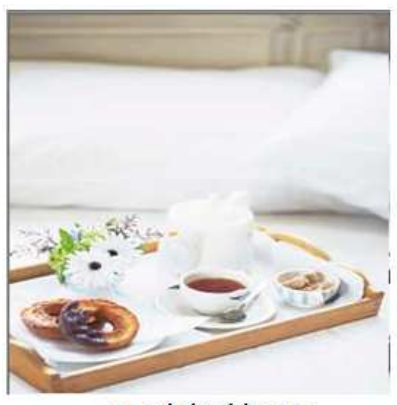

a- original image

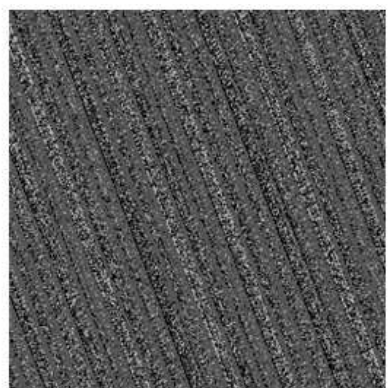

b- ciphered $C_{b}$ image
Figure (18). Scrambling $C_{b}$ image.

$$
\begin{aligned}
& {\left[\begin{array}{l}
x^{\prime} \\
y^{\prime}
\end{array}\right]=\left[\begin{array}{cc}
8 & 13 \\
21 & 34
\end{array}\right]\left[\begin{array}{l}
0 \\
0
\end{array}\right][\bmod 3]=(0,0),} \\
& {\left[\begin{array}{l}
x^{\prime} \\
y^{\prime}
\end{array}\right]=\left[\begin{array}{cc}
8 & 13 \\
21 & 34
\end{array}\right]\left[\begin{array}{l}
0 \\
1
\end{array}\right][\bmod 3]=(1,1)}
\end{aligned}
$$

Figure (16). Scrambling Y image. 


\begin{tabular}{|l|l|l|}
\hline 76 & 103 & 45 \\
\hline 38 & 75 & 80 \\
\hline 104 & 105 & 88 \\
\hline
\end{tabular}

a. original image

Figure (19). Results after scrambling $C_{b}$ image.

\subsubsection{2. $C_{r}$ Band}

In this band, the cipher used Fibonacci-Lucas transform for scrambling all pixels in this band by applying equation (9) as illustrated in figure (20) as well as in calculating the new ciphered values for supposed example as shown in figure (21).

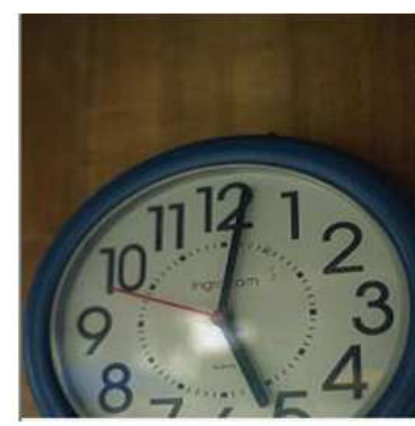

a- original image

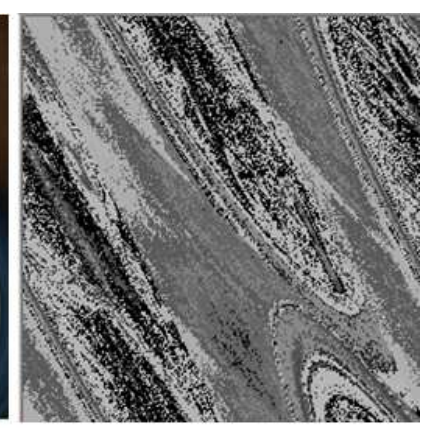

b- ciphered $C_{r}$ image
Figure (20). Scrambling $C_{r}$ image.

$$
\begin{aligned}
& {\left[\begin{array}{l}
x^{\prime} \\
y^{\prime}
\end{array}\right]=\left[\begin{array}{ll}
1 & 1 \\
2 & 1
\end{array}\right]\left[\begin{array}{l}
0 \\
0
\end{array}\right][\bmod 3]=(0,0),} \\
& {\left[\begin{array}{l}
x^{\prime} \\
y^{\prime}
\end{array}\right]=\left[\begin{array}{ll}
1 & 1 \\
2 & 1
\end{array}\right]\left[\begin{array}{l}
0 \\
1
\end{array}\right][\bmod 3]=(1,1)}
\end{aligned}
$$

\begin{tabular}{|l|l|l|}
\hline 97 & 36 & 31 \\
\hline 119 & 175 & 103 \\
\hline 80 & 170 & 65 \\
\hline
\end{tabular}

a. $Y$ image

\begin{tabular}{|l|l|l|}
\hline 97 & 65 & 175 \\
\hline 103 & 36 & 80 \\
\hline 170 & 119 & 31 \\
\hline
\end{tabular}

d. final scrambling $Y$ image

\begin{tabular}{|l|l|l|}
\hline 76 & 75 & 88 \\
\hline 104 & 103 & 80 \\
\hline 38 & 105 & 45 \\
\hline
\end{tabular}

b. $C_{b}$ image

\begin{tabular}{|l|l|l|}
\hline 76 & 45 & 103 \\
\hline 80 & 75 & 38 \\
\hline 105 & 104 & 88 \\
\hline
\end{tabular}

e. final scrambling $C b$ image

\begin{tabular}{|l|l|l|}
\hline 113 & 170 & 86 \\
\hline 93 & 109 & 102 \\
\hline 58 & 181 & 142 \\
\hline
\end{tabular}

a. original image

Figure (21). Results after scrambling $C_{r}$ image

\subsubsection{Scrambling All Pixels in Colored Image}

In this step, the cipher used Arnold transform for scrambling all pixels in this band by applying equation (4) as illustrated in figure (22) as well as in calculating the new ciphered values for supposed example as shown in figure (23).

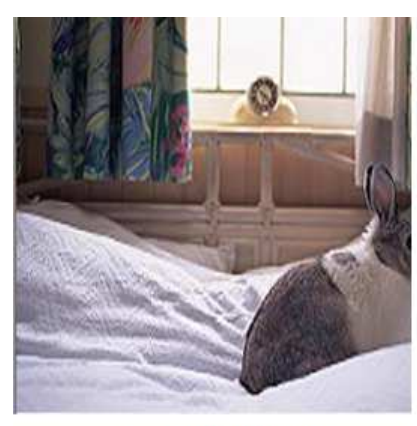

a- original image

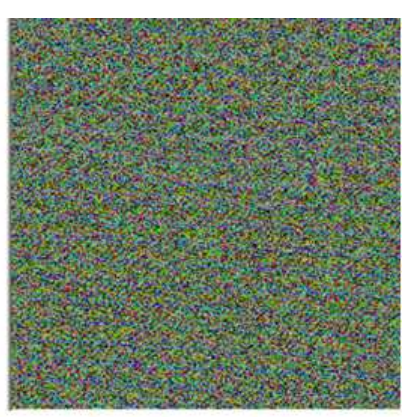

b- ciphered colored image
Figure (22). Scrambling Colored Image.

$$
\begin{aligned}
& {\left[\begin{array}{l}
x^{\prime} \\
y^{\prime}
\end{array}\right]=\left[\begin{array}{cc}
20 & 19 \\
1 & 1
\end{array}\right]\left[\begin{array}{l}
0 \\
0
\end{array}\right][\bmod 3]=(0,0),} \\
& {\left[\begin{array}{l}
x^{\prime} \\
y^{\prime}
\end{array}\right]=\left[\begin{array}{cc}
20 & 19 \\
1 & 1
\end{array}\right]\left[\begin{array}{l}
0 \\
1
\end{array}\right][\bmod 3]=(1,1)}
\end{aligned}
$$

\begin{tabular}{|l|l|l|}
\hline 113 & 102 & 181 \\
\hline 142 & 170 & 93 \\
\hline 109 & 58 & 86 \\
\hline
\end{tabular}

c. $C_{r}$ image

\begin{tabular}{|l|l|l|}
\hline 113 & 86 & 170 \\
\hline 93 & 102 & 109 \\
\hline 58 & 142 & 181 \\
\hline
\end{tabular}

f. final scrambling $C_{r}$ image

Figure (23). Scrambling colored image.

\subsection{Second Stage}

The input for this stage is the final ciphered image that result from first stage and this image will be passed through the same processes in the first stage but in reverse ordering as follows: 


\subsubsection{Applying Inverse Arnold Transform}

In this step, the inverse Arnold transform will be applied in colored image as illustrated in figure (24).

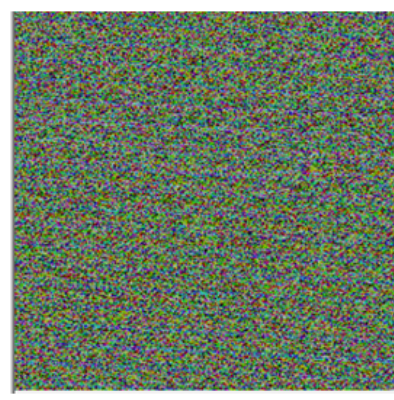

a- ciphered image

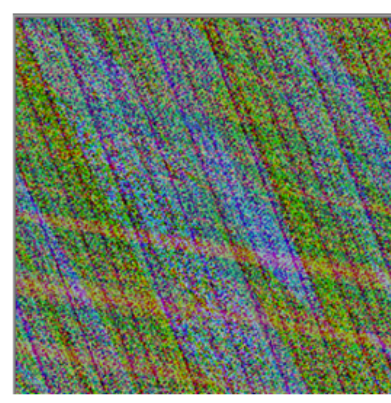

b- ciphered image after applying Arnold transform
The inverse Arnold transform can be illustrated in figure (25) by using equation below:

Figure (24). Applying inverse Arnold transform on colored image.

\begin{tabular}{|l|l|l|}
\hline 97 & 65 & 175 \\
\hline 103 & 36 & 80 \\
\hline 170 & 119 & 31 \\
\hline
\end{tabular}

\begin{tabular}{|l|l|l|}
\hline 76 & 45 & 103 \\
\hline 80 & 75 & 38 \\
\hline 105 & 104 & 88 \\
\hline
\end{tabular}

\begin{tabular}{|l|l|l|}
\hline 113 & 86 & 170 \\
\hline 93 & 102 & 109 \\
\hline 58 & 142 & 181 \\
\hline
\end{tabular}

a. bands of ciphered image.

\begin{tabular}{|l|l|l|}
\hline 97 & 36 & 31 \\
\hline 110 & 175 & 103 \\
\hline 80 & 170 & 65 \\
\hline
\end{tabular}

\begin{tabular}{|l|l|l|}
\hline 76 & 75 & 88 \\
\hline 104 & 103 & 80 \\
\hline 38 & 105 & 45 \\
\hline
\end{tabular}

\begin{tabular}{|l|l|l|}
\hline 113 & 102 & 181 \\
\hline 142 & 170 & 93 \\
\hline 109 & 58 & 86 \\
\hline
\end{tabular}

b. bands after applying inverse Arnold transform.

Figure (25). Applying inverse Arnold transform on colored image.

\subsubsection{Return Pixels in Each Band to Its State before Scrambling}

As illustrated in proposed system block diagram, each band will be decryption with specific inverse transform, so this step will be separated into three states.

\subsubsection{Y Band}

In this band, the decipher used inverse Arnold transform for return all pixels in this band to its past original state before scrambling as illustrated in figure (26).
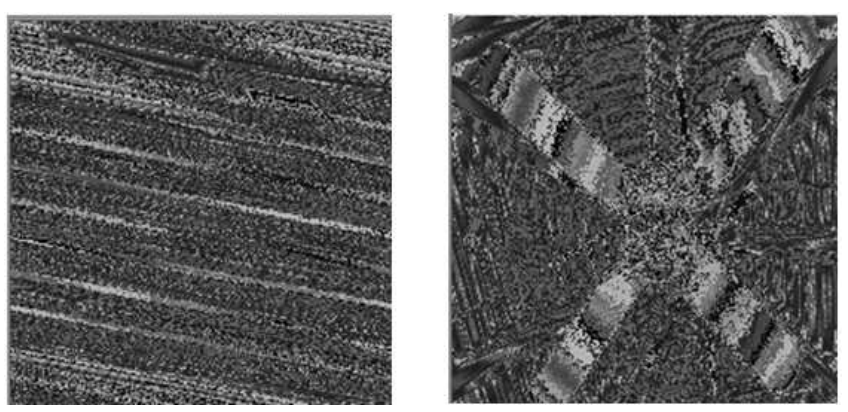

Figure (26). Applying inverse Arnold transform on Y image.
Calculating values before scrambling in $\mathrm{Y}$ band for supposed example will be shown in follows and in figure (27).

$$
\begin{aligned}
& {\left[\begin{array}{l}
x^{\prime} \\
y^{\prime}
\end{array}\right]=\left[\begin{array}{cc}
1 & -5 \\
-1 & 6
\end{array}\right]\left[\begin{array}{l}
0 \\
0
\end{array}\right][\bmod 3]=(0,0),} \\
& {\left[\begin{array}{l}
x^{\prime} \\
y^{\prime}
\end{array}\right]=\left[\begin{array}{cc}
1 & -5 \\
-1 & 6
\end{array}\right]\left[\begin{array}{l}
0 \\
1
\end{array}\right][\bmod 3]=(1,0)}
\end{aligned}
$$

\begin{tabular}{|l|l|l|}
\hline 97 & 36 & 31 \\
\hline 119 & 175 & 103 \\
\hline 80 & 170 & 65 \\
\hline
\end{tabular}

\begin{tabular}{|l|l|l|}
\hline 97 & 102 & 103 \\
\hline 36 & 65 & 119 \\
\hline 31 & 80 & 175 \\
\hline
\end{tabular}

Figure (27). Applying inverse Arnold transform on Y image.

\subsubsection{2. $C_{b}$ Band}

In this band, the decipher used inverse Fibonacci transform for return all pixels in this band to its past original state before scrambling as illustrated in figure (28). 

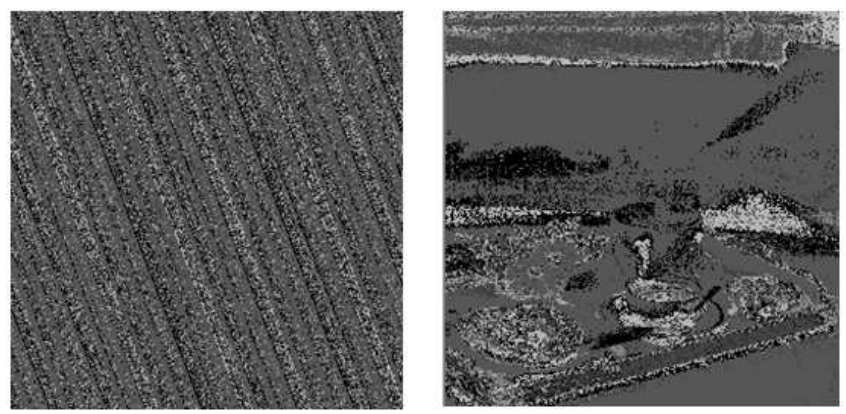

Figure (28). Applying inverse Fibonacci transform on $C_{b}$ image.

Calculating values before scrambling in $\mathrm{Cb}$ band for supposed example will be shown in follows and in figure (29).

$$
\begin{aligned}
& {\left[\begin{array}{l}
x^{\prime} \\
y^{\prime}
\end{array}\right]=\left[\begin{array}{cc}
-34 & 13 \\
21 & -8
\end{array}\right]\left[\begin{array}{l}
0 \\
0
\end{array}\right][\bmod 3]=(0,0),} \\
& {\left[\begin{array}{l}
x^{\prime} \\
y^{\prime}
\end{array}\right]=\left[\begin{array}{cc}
-34 & 13 \\
21 & -8
\end{array}\right]\left[\begin{array}{l}
0 \\
1
\end{array}\right][\bmod 3]=(1,0)}
\end{aligned}
$$

\begin{tabular}{|l|l|l|}
\hline 76 & 75 & 88 \\
\hline 104 & 103 & 80 \\
\hline 38 & 105 & 45 \\
\hline
\end{tabular}

\begin{tabular}{|l|l|l|}
\hline 76 & 103 & 45 \\
\hline 38 & 75 & 80 \\
\hline 104 & 105 & 88 \\
\hline
\end{tabular}

Figure (29). Applying inverse Fibonacci transform on $C_{b}$ image.

\subsubsection{3. $C_{r}$ Band}

In this band, the decipher used inverse Fibonacci-Lucas transform for return all pixels in this band to its past original state before scrambling as illustrated in figure (30).
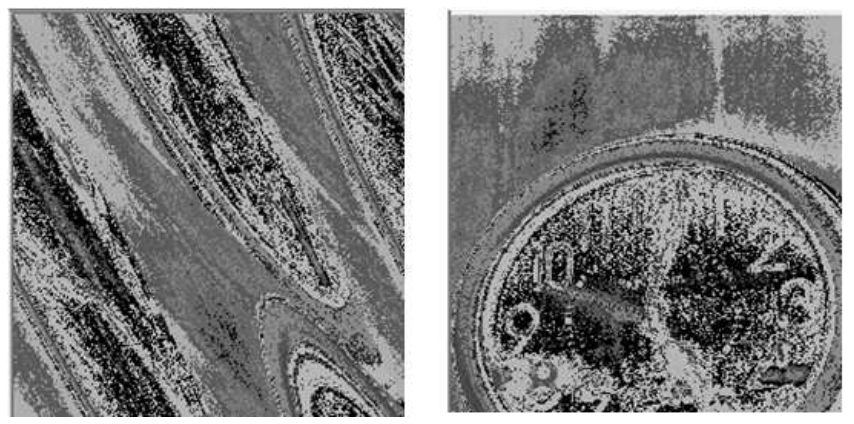

Figure (30). Applying inverse Fibonacci-Lucas transform on $C_{r}$ image.

Calculating values before scrambling in $\mathrm{Cr}$ band for supposed example will be shown in follows and in figure (31).

$$
\begin{aligned}
& {\left[\begin{array}{l}
x^{\prime} \\
y^{\prime}
\end{array}\right]=\left[\begin{array}{cc}
-1 & 1 \\
2 & -1
\end{array}\right]\left[\begin{array}{l}
0 \\
0
\end{array}\right][\bmod 3]=(0,0),} \\
& {\left[\begin{array}{l}
x^{\prime} \\
y^{\prime}
\end{array}\right]=\left[\begin{array}{cc}
-1 & 1 \\
2 & -1
\end{array}\right]\left[\begin{array}{l}
0 \\
1
\end{array}\right][\bmod 3]=(1,2)}
\end{aligned}
$$

\begin{tabular}{|l|l|l|}
\hline 113 & 102 & 181 \\
\hline 142 & 170 & 93 \\
\hline 109 & 58 & 86 \\
\hline
\end{tabular}

\begin{tabular}{|l|l|l|}
\hline 113 & 170 & 86 \\
\hline 93 & 109 & 102 \\
\hline 58 & 181 & 142 \\
\hline
\end{tabular}

Figure (31). Applying inverse Fibonacci-Lucas transform on $C_{r}$ image.

\subsubsection{Public Key Decryption}

As illustrated in proposed system block diagram, each band will be deciphered with different key or keys, so this step will be separated into three states.

\subsubsection{Y Band}

In this band, the decipher used received private key for decryption all pixels in this band by applying equation (2) as illustrated in figure (32) as well as in calculating the new ciphered values for supposed example with key $(107,187)$ as shown in figure (33).
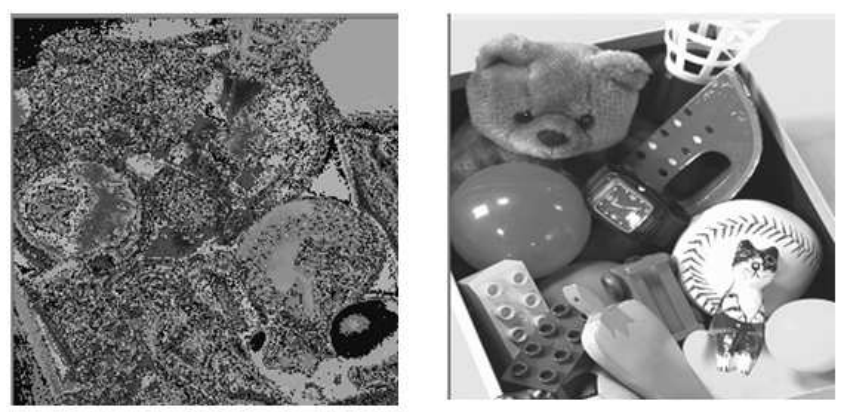

Figure (32). Decryption Y image.

$$
P_{(113)}=(113)^{107} \bmod 187=113
$$

\begin{tabular}{|l|l|l|}
\hline 113 & 170 & 86 \\
\hline 93 & 109 & 102 \\
\hline 58 & 181 & 142 \\
\hline
\end{tabular}

\begin{tabular}{|l|l|l|}
\hline 97 & 102 & 103 \\
\hline 36 & 65 & 119 \\
\hline 31 & 80 & 175 \\
\hline
\end{tabular}

Figure (33). Results after applying key $(107,187)$ on Y image.

\subsubsection{2. $C_{b}$ Band}

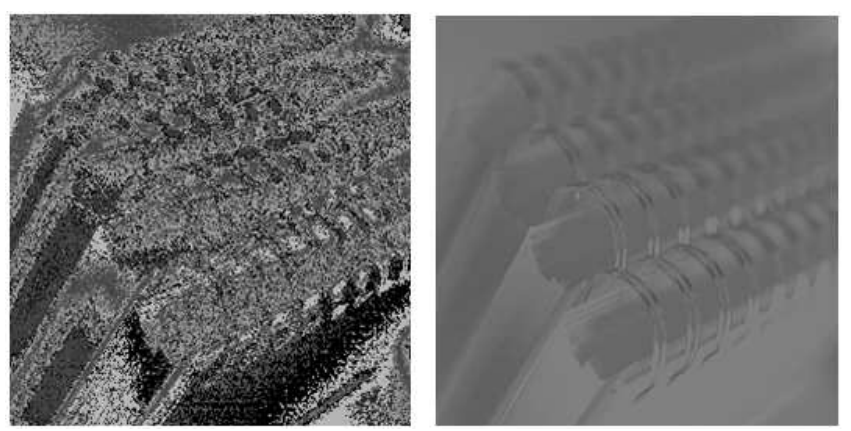

Figure (34). Decryption $C_{b}$ image.

In this band, the decipher used sender public key and received private key for decryption all pixels in this band 
by applying equation (2) with additional inverse normalization process as illustrated in figure (34) as well as in calculating the new deciphered values for supposed example with keys $(107,187)$ and $(11,133)$ as shown in figure (35).

$P(76)=(76)^{11} \bmod 133=76$ then normalized all values to cipher with key $(11,133)$

\begin{tabular}{|l|l|l|}
\hline 76 & 103 & 45 \\
\hline 38 & 75 & 80 \\
\hline 104 & 105 & 88 \\
\hline
\end{tabular}

a. ciphered image

\begin{tabular}{|l|l|l|}
\hline 76 & 31 & 68 \\
\hline 19 & 94 & 54 \\
\hline 62 & 14 & 65 \\
\hline
\end{tabular}

b. first deciphered image

$$
\begin{gathered}
76 \times(255 / 132)=147 \\
P(147)=(147)^{107} \bmod 187=76
\end{gathered}
$$

\begin{tabular}{|l|l|l|}
\hline 147 & 59 & 132 \\
\hline 37 & 181 & 105 \\
\hline 120 & 28 & 125 \\
\hline
\end{tabular}

c. normalized image

\begin{tabular}{|l|l|l|}
\hline 148 & 104 & 55 \\
\hline 126 & 80 & 41 \\
\hline 120 & 63 & 5 \\
\hline
\end{tabular}

c. final deciphered image

Figure (35). Results deciphering $C_{b}$ image.

\subsubsection{3. $C_{r}$ Band}

In this band, the decipher used sender public key for decryption all pixels in this band by applying equation (2) as illustrated in figure (36) as well as in calculating the new ciphered values for supposed example with key $(107,187)$ as shown in figure (37).

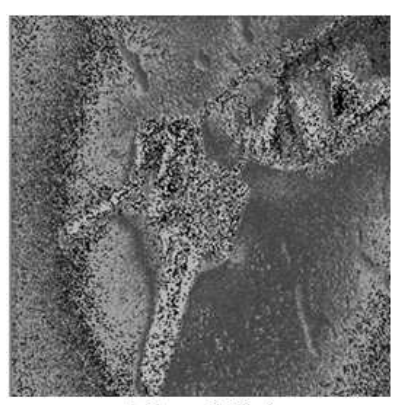

a- ciphered $\mathrm{Cr}$ image

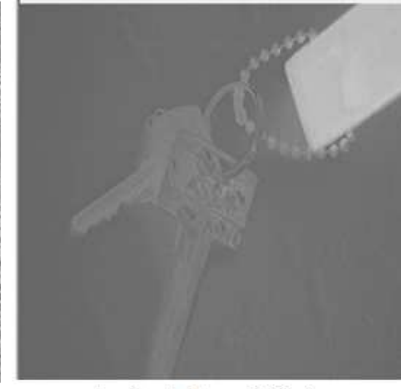

b- deciphered $\mathrm{Cr}$ image

Figure (36). Decryption $C_{r}$ image.

\begin{tabular}{|l|l|l|}
\hline 97 & 102 & 103 \\
\hline 36 & 65 & 119 \\
\hline 31 & 80 & 175 \\
\hline
\end{tabular}

\begin{tabular}{|l|l|l|}
\hline 148 & 104 & 55 \\
\hline 126 & 80 & 41 \\
\hline 120 & 63 & 5 \\
\hline
\end{tabular}

a. bands after normalization.

\begin{tabular}{|l|l|l|}
\hline 133 & 140 & 141 \\
\hline 49 & 89 & 163 \\
\hline 43 & 109 & 240 \\
\hline
\end{tabular}

\begin{tabular}{|l|l|l|}
\hline 203 & 134 & 75 \\
\hline 173 & 109 & 56 \\
\hline 164 & 87 & 7 \\
\hline
\end{tabular}

\begin{tabular}{|l|l|l|}
\hline 113 & 170 & 86 \\
\hline 93 & 109 & 102 \\
\hline 58 & 181 & 142 \\
\hline
\end{tabular}

\begin{tabular}{|l|l|l|}
\hline 97 & 102 & 103 \\
\hline 36 & 65 & 119 \\
\hline 31 & 80 & 175 \\
\hline
\end{tabular}

Figure (37). Results after applying key $(107,187)$ on $C_{r}$ image.

\subsubsection{Return Original Values before Normalization}

In this step inverse normalized ratio will be applied to each pixel in each band as follows:

For y band: pixel value $\times 255 / 186$, for $\mathrm{Cr}$ band: pixel value $\times 255 / 132$, and for

$\mathrm{Cb}$ band: pixel value $\times 255 / 186$. this process results will be illustrated in figure (38).

\begin{tabular}{|l|l|l|}
\hline 24 & 94 & 98 \\
\hline 65 & 76 & 104 \\
\hline 71 & 87 & 78 \\
\hline
\end{tabular}

\begin{tabular}{|l|l|l|}
\hline 47 & 182 & 189 \\
\hline 126 & 147 & 201 \\
\hline 137 & 169 & 150 \\
\hline
\end{tabular}

b. bands before normalization.

Figure (38). Applying inverse normalization process. 


\subsubsection{Translating to BMP Image}

To translate an image from $\mathrm{YCbCr}$ format to BMP format, inverse array used in equation (10) will be used as follows in equation (11) the result of applying this equation will be

$$
\left[\begin{array}{c}
\text { red } \\
\text { green } \\
\text { blue }
\end{array}\right]=\left[\begin{array}{ccc}
0.9986 & -0.0004 & 1.4027 \\
1.0011 & -0.3439 & -0.7142 \\
0.9982 & 1.7722 & -0.0015
\end{array}\right]\left[\begin{array}{c}
Y-16 \\
C_{b}-128 \\
C_{r}-128
\end{array}\right]
$$
illustrated in figure (39).

\begin{tabular}{|l|l|l|}
\hline 133 & 140 & 141 \\
\hline 49 & 89 & 163 \\
\hline 43 & 109 & 240 \\
\hline
\end{tabular}

a. $Y$ band

\begin{tabular}{|l|l|l|}
\hline 3 & 200 & 210 \\
\hline 30 & 100 & 250 \\
\hline 40 & 150 & 255 \\
\hline
\end{tabular}

d. red band

\begin{tabular}{|l|l|l|}
\hline 203 & 143 & 75 \\
\hline 173 & 109 & 56 \\
\hline 164 & 87 & 7 \\
\hline
\end{tabular}

b. $C_{b}$ band

\begin{tabular}{|l|l|l|}
\hline 150 & 80 & 100 \\
\hline 22 & 66 & 120 \\
\hline 8 & 78 & 250 \\
\hline
\end{tabular}

e. green band

\begin{tabular}{|l|l|l|}
\hline 47 & 182 & 189 \\
\hline 126 & 147 & 201 \\
\hline 137 & 169 & 150 \\
\hline
\end{tabular}

c. $C_{r}$ band

\begin{tabular}{|l|l|l|}
\hline 250 & 150 & 30 \\
\hline 100 & 40 & 20 \\
\hline 90 & 20 & 10 \\
\hline
\end{tabular}

f. blue band

Figure (39). Translating $Y C_{b} C_{r}$ image into BMP image.

\section{Results}

After applying the proposed system on colored image, the following results will be founded.

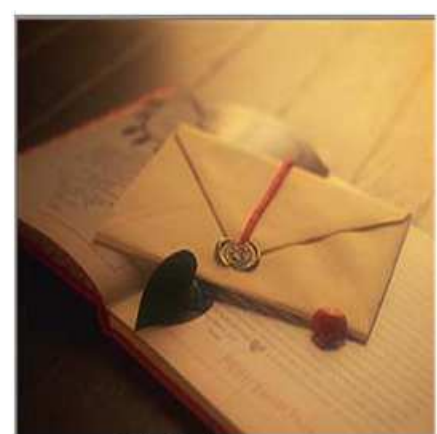

a- BMP image

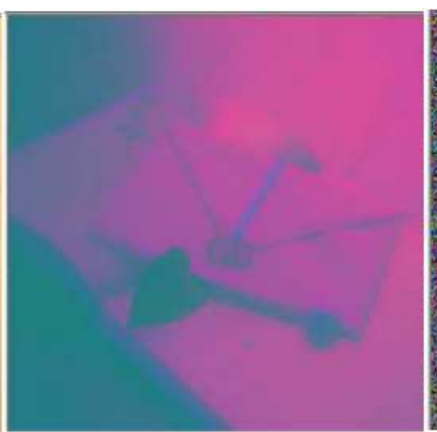

b- $Y C_{b} C_{r}$ image

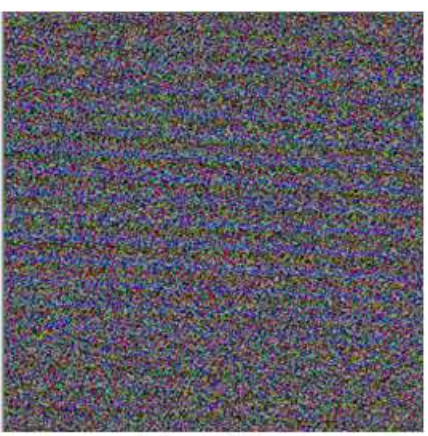

c- ciphered image

Figure (40). Result (1) of the proposed system.

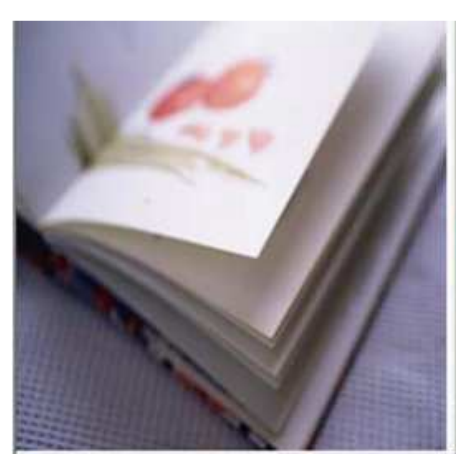

a- BMP image

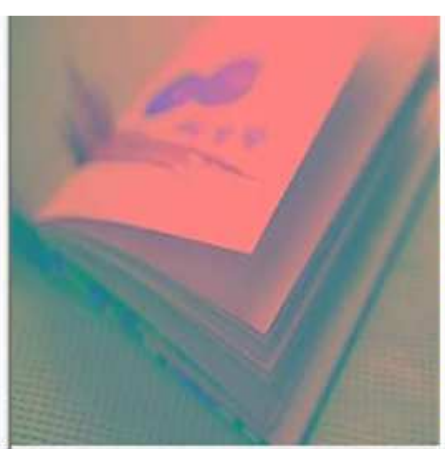

b- $Y C_{b} C_{r}$ image

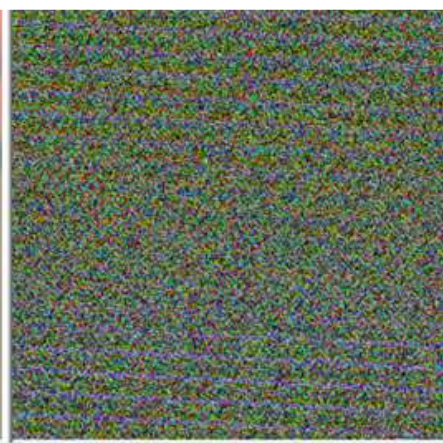

c- ciphered image

Figure (41). Result (2) of the proposed system. 


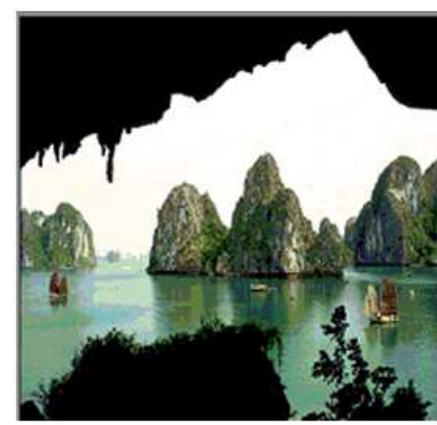

a- BMP image

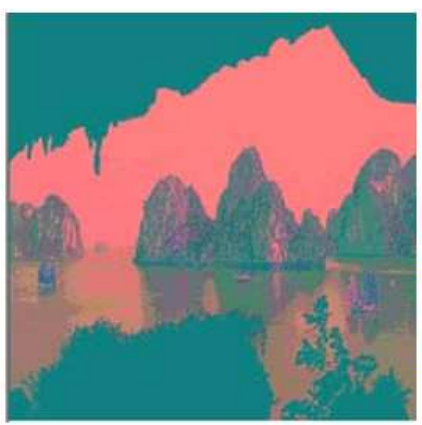

b- $Y C_{b} C_{r}$ image

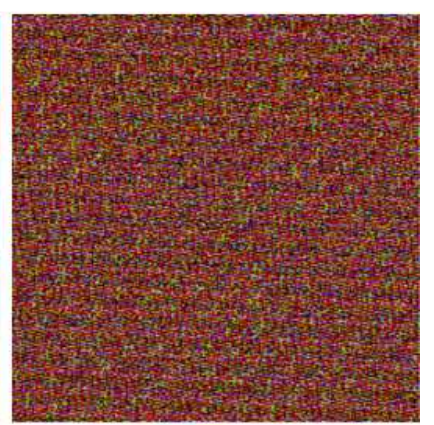

c- ciphered image

Figure (42). Result (3) of the proposed system

\section{Conclusion}

From the above discussions and results we conclude that:

- Scrambling image pixel is a powerful tool in ciphering image and it make image unreadable and unproductive, but scrambling methods must have long randomness cycle to avoid repeated values of new position for all pixels in original image.

- Public key encryption changes the pixels value in image in fashion that make the image can be predictive with huge amount of noise, so this method cannot be used alone.

- Normalization process is an important step to make information space identical to key space without losing information that damage image.

\section{References}

[1] Linhua Zhang, Xiaofeng Liao , Xuebing Wang " An Image Encryption Approach Based on Chaotic Maps", .elsevier , China, 2004.

\section{Biography}

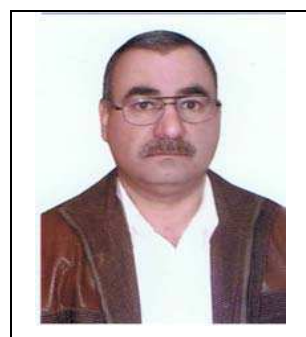

Dr. Salman Abd Kadum, Ph. D., Ms.C. Technical Education Foundation, Najaf Technical Institute, Najaf, IRAQ. He received B.Sc. in Physics sciences (1983) from Baghdad university, High Deploma in computer science (1988) from training \& research institute, Baghdad, Iraq, M.Sc. in computer sciences (1998) from Gajah Mada university, Indonesia, and $\mathrm{Ph}$. D. in computer science its also from Gajah Mada University, Indonesia (2004), worked as a lecturer as long time and a head of computer system in Najaf Technical Institute for two years, he is also a master of computer center for two years, and he is also as lecturer for eight years and a head of software engineering in Al-Imam Jaafar Alsadeq university for five years, he has many published papers. His researches interests are in image processing, and signal processing.
[2] Kwok-Wo Wong, Bernie Sin-Hung Kwok, and Wing-Shing Law " A Fast Image Encryption Scheme based on Chaotic Standard Map", HONG KONG, 2006.

[3] Abdullah Sharaf Alghamdi, and Hanif Ullah "A Secure Iris Image Encryption Technique Using Bio-Chaotic Algorithm", IJCNS, 2010.

[4] Minati Mishra, Priyadarsini Mishra, M.C. Adhikary and Sunit Kumar," Image Encryption Using Fibonacci-Lucas Transformation", IJCIS, India, 2012.

[5] Rajinder Kaur, Er.Kanwalprit Singh " Image Encryption Techniques: A Selected Review", IOSR-JCE, India, 2013.

[6] Quist-Aphetsi Kester " Image Encryption Based on the RGB PIXEL Transposition and Shuffling" I. J. Computer Network and Information Security, 2013.

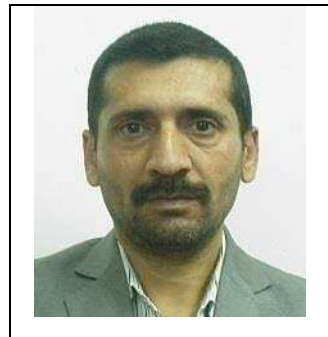

ALI ADUL AZEEZ MOHAMMAD BAKER University of Kufa, Education College, Najaf, IRAQ. He received B.Sc. in computer sciences (2010), civil engineering (1990), and M.Sc. in computer sciences (2012), worked as a teacher in Kufa University, has many published papers. His research interests are in image processing, and security (biometrics, and steganography), He's Associate teacher in Computer Science at the University of Kufa - Najaf, IRAQ. 